\title{
Use of vulture bone flutes and other products in China
}

\author{
Roller MaMing*, Kewei Jiang, Junwei Li, Li Chen, Gang Lei
}

Xinjiang Institute of Ecology and Geography, Chinese Academy of Sciences

Beijing Road, Urumqi, 830011, Xinjiang

P. R. of China

*Corresponding author: maming@ms.xjb.ac.cn

http://dx.doi.org/10.4314/vulnew.v78i1.3

\section{Introduction}

Vulture bone flutes and whistles not only play wonderful music, but they also have a certain collection value as arts and crafts gifts. Additionally, in some parts of China, vulture bones are used to make beads, bracelets, necklaces, cigarette sticks, and even mascots or amulets to drive away evil spirits. There is a wide variety of products with vulture bones found in China.

A very old bone flute was unearthed in Jiahu, Henan Province in 1986, which became an important discovery and caused great surprise when estimates placed the age of the flute as approximately 9,000 years old (Zhang et al. 1999). Other bone flutes or bone whistles with a long history have been excavated in many other places in the East (Wang 2001), and this traditional use of vulture bones remains today; it is deeply rooted in Chinese culture. Once considered unique compared to other parts of the world, the use of vulture bone flutes and whistles continues to be praised as a "national treasure" or a world cultural heritage.

However, the more recent discovery of a bone flute in Germany caused significant surprise: the roughly $22 \mathrm{~cm}$ long five-hole-flute made from the radius bone of a Eurasian Griffon (Gyps fulvus) was estimated to be approximately 35,000 years old (Bower, 2009). Similar findings of bone flutes and whistles have also been found in Slovenia, France, Mexico, Peru and other countries, although not all of these are made from vulture bones.

As the gradual prosperity of people's material life in China has increased, more and more people have begun to consume vulture products, which is a very complicated and difficult situation to address. Apart from bone flutes and whistles, our own investigations have revealed that many other vulture products such as meat, viscera and feathers are used in China, such as for traditional Chinese medicine, feather fans, specimens, gifts, amulets, food ingredients, etc. (Ma et al. 2017). Here we report further on these findings.

\section{Methods}

During fieldwork for two research projects on Himalayan Vulture (Gyps himalayensis) and Black Vulture (Aegypius monachus) between 2012 and 2019 (Ma 2019), we also carried out on-site 
investigations in Xinjiang, Qinghai, Tibet, Yunnan, Sichuan, Gansu and other places where vulture products were found. These places included venues such musical instrument stores, markets or bazaars, museums, specimen exhibitions, schools, rural workshops, literature, art and concert performances, festivals, horse racing and places for sheep competitions. We aimed to understand the source of vulture products, what species are used, sizes, ages, uses, quantities, prices, trade status, legal attitude of people and any government regulations.

In addition, through internet searches and telephone contacts, we obtained other information relevant to the use of vulture body parts such as product catalogues, personal collection, photographic evidence, case information published by local police stations and various media and news stories. The website search used a variety of key words in both Chinese (鹰骨笛、熟骨笛、雕 翼笛、骨笛和鹰笛) and English (bone flute \eagle bone flute \eagle's wing flute \vulture bone flute). We used Excel and SPSS to collate, analyse and map the data.

\section{Results and Discussion}

Most of the bone flutes being sold at present are made from the wing bones of vultures. The flutes are generally $24-26 \mathrm{~cm}$ long and about $1.5 \mathrm{~cm}$ in diameter. There are three to eight holes in the bone flute - the Tajik bone flute from Xinjiang usually only has three holes (Han 2010), gradually turning into four and then eight holes towards the east and south of China (Zhang 1990, Xue 2010). Tibet's bone flute is relatively complex and diverse, with five, six and seven scales, and even able to play ethnic minority music or foreign music rich in changing sounds.

\section{(1) Origin and supply:}

The source for the bones is mainly in the plateau area in the western part of China, such as Tibet (not only in Lhasa, Ci \& Sun 2019), Sichuan, Yunnan, Qinghai (Xue 2010), Gansu (Duan 1995) and Xinjiang. Each province has at least ten counties (Figure 1). Because of the different bone flute styles, there are two main areas that generally supply the whole country: one is Tajik's 3-hole style, the other is Tibetan's 6-hole style. Of course, there are several neighboring or nearby countries also with different styles, such as Afghanistan, Tajikistan, Uzbekistan, Iran, etc. Coincidentally, Tibetan, Tajik, Kirgiz and other ethnic distribution areas in the west of China coincide with the natural distribution of vultures. How suppliers actually obtain the raw bone materials is very secretive, and although there are high profits available to hunters, there are high risks. However, accurate hunting information is very difficult to obtain.

\section{(2) Sales Location and Network:}

Sale locations are all over the country, such as in Shanghai, Beijing, Chengdu, Lhasa, Chongqing, Shenyang, Urumqi, Kashgar (many locations), Tashkurgan, Shache, Zepu, Yecheng, Pishan, Wuqia, Aktau, Atush, etc. The sales network covers almost $79 \%$ of the country, including 27 provincial administrative regions and more than a hundred cities. In Hohhot, Inner Mongolia, the name of a shop, "Olunchun Cultural Heritage" displays the banner of intangible cultural heritage, making and selling new bone flutes, which are 
made of Griffon Vulture ulna bones.

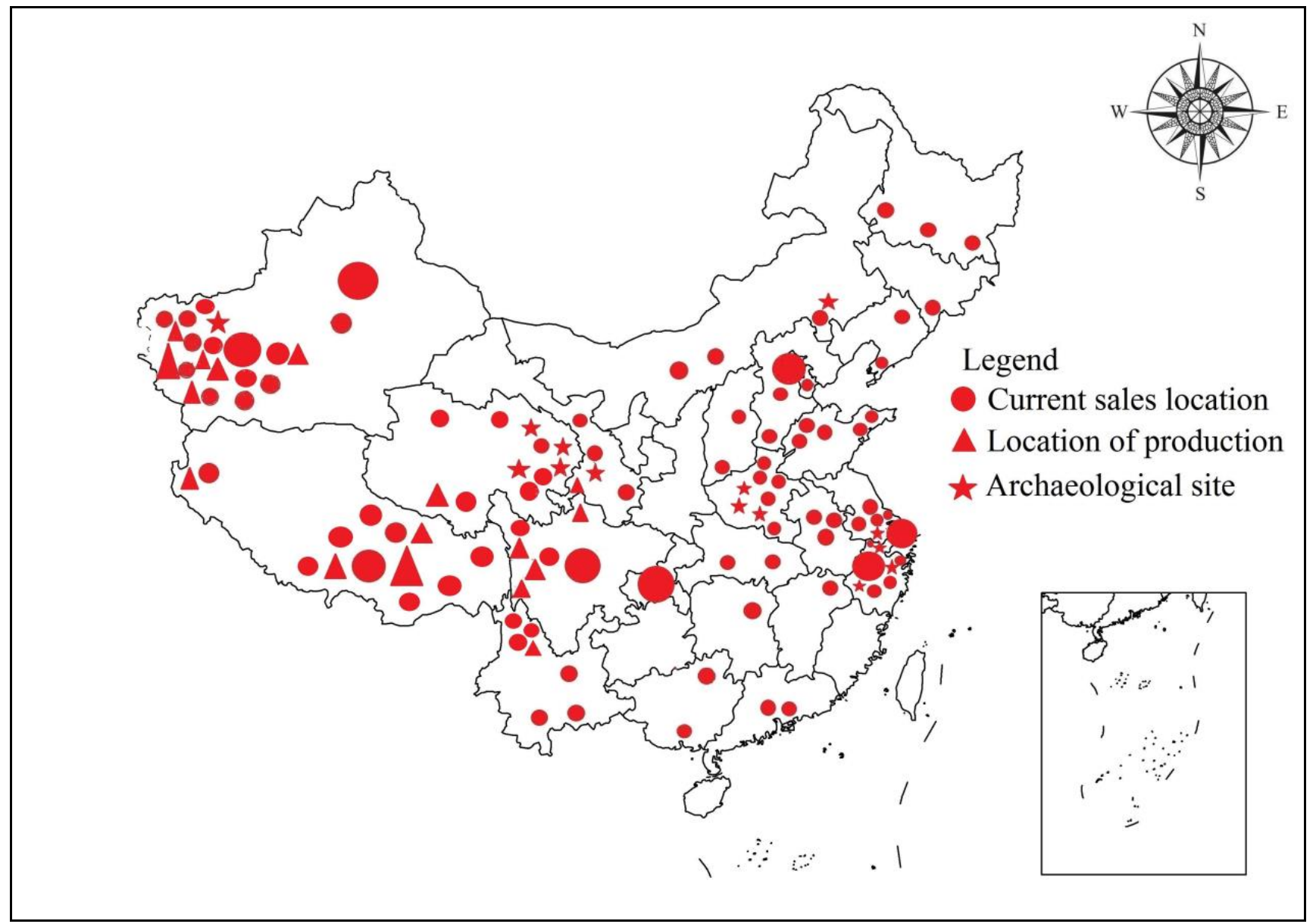

Figure 1: The production site (triangle), sales site (circle) and archaeological discovery site (pentagram) of vulture wing bone flute in China

(3) Cultural Tradition and Vulture Product Type:

According to our preliminary statistics, 11-15 kinds of vulture products are available in China. These product include not just bone flutes, but bone whistles, bone bead strings (necklaces, bracelets, etc.), cigarette holders or tobacco pots (cigarette rods), feather fans, whistles made of vulture feather tubes, Chinese medicine components (see Compendium of Materia Medica by Dr. Li Shizhen, a doctor of Ming Dynasty), Tibetan needle boxes made Griffon Vulture bones, and food materials, etc. (Table 1). The trading, acquisition and identification of vulture products includes living individuals, corpses, bone raw materials, beads, bone tubes, feather tubes, feet, wings, claws, beaks and skulls. The monthly renewal of products in online stores accounts for more than $90 \%$ of the trade we observed and most products are sold quickly through online auctions, with large sales volumes. In addition to vulture parts, there are products of other large raptors, such as those from Lammergeier (Bearded Vulture; Gypaetus barbatus), Golden Eagle (Aquila chrysaetos) and Eagle Owl (Bubo bubo) (MaMing et al. 2014). 
Table 1: Origin and price of vulture products in China (USD)

\begin{tabular}{llrlll}
\hline Product category & Origin & No & Price $\mathbf{( \$ )}$ & Access/Sources & Sales method \\
\hline Tibetan bone flute & Tibet, etc. & 91 & $1000-9000$ & Poison, other & Network, store, market \\
\hline Tajik vulture flute & Xinjiang & 87 & $1500-6000$ & Poison, natural death & Shop, home, internet, \\
bazaar
\end{tabular}

China is an ancient civilization, and there are many archaeological sites with vulture product remains, such as Jiahu bone flutes in Henan and Hemudu bone whistles in Zhejiang (Song 2007). Vulture products are also found in Jiangsu, Qinghai, Gansu, Bachu in Xinjiang, and Xinglongwa culture in Chifeng, Inner Mongolia. From ancient times, the so-called "bone grafting secret recipe" (Li Shizhen, 1981) for the treatment of injuries caused by falls has been adopted for more recent use.

\section{(4) Sales methods:}

There are diversified business models for the sale of vulture parts in China, such as physical stores, bazaars and rural markets, private farmer pre-sales, online ordering (that only accept bookings and the processing period is about one week), exhibitions and sales conferences and workshop sales - all have postal express delivery. Griffon Vulture bone flutes frequently appear at various occasions such as tourist attractions, weddings, folk competitions (games), festival parties, TV programs and so on. Sometimes these items are directly sold to tourists after performances and exhibitions at scenic spots, which is very hard to track and easy to conceal from authorities. The most expensive bone flutes can be sold for tens of thousands of US dollars. A Griffon Vulture bone flute in Beijing can be sold for 300000 yuan (1 US dollar $=6.3$ yuan), and a feather fan can be sold for thousands of yuan (see photo screenshot). Even after artificial aging, many flutes are sold as antiques at a higher price. We estimate that about $75 \%$ of bone flutes are sold as ancient wing bone flutes after they are deliberately made to look old.

\section{(5) Amount of Product Data on the Internet:}

There are many sales posts on a variety of websites, and the number of available vulture products is amazing (Table 2). There are dozens of known sales websites and 200,000-300,000 
promotional posts, which are difficult to track for authorities. Obviously, with the use of information networking and the relatively low cost, money can be made quickly. From the increasing number of poaching cases every year (MaMing et al., 2015), the scale of market demand is clear. However, some public uses of vulture parts are obvious and others are hidden. For example, the demand for bone flutes can reach a peak through its visibility via a range of media, but a large number of private demands, such as the purchase of feather fans, parts for medicinal materials and food materials are often invisible used privately.

Table 2: Statistical record of online sales of vulture products in China

\begin{tabular}{|c|c|c|c|c|c|c|c|}
\hline $\begin{array}{l}\text { Website } \\
\text { Product }\end{array}$ & XianyuNet & TaoBao & Alibaba & WeChat & Weipaitang & Tieba/Baidu & Total \\
\hline Bone material & 23 & & & 2 & & 142 & 167 \\
\hline Bone flute (Old) & 9 & & & 14 & & 4 & 27 \\
\hline Bone flute (New) & 5 & & & 2 whistles & & 4 & 11 \\
\hline Bead string (Old) & 6 & & & 1 & & 13 & 20 \\
\hline Bead string (New) & 18 & 1 & 5 & 6 & & 237 & 267 \\
\hline Bone pipe & 16 & & & 1 & 1 & 2 & 20 \\
\hline Claw & 3 & & & 16 & & 79 & 98 \\
\hline Head (Skull) & 2 & & & 1 & & 21 & 24 \\
\hline Feather & & 5 shops & 3 shops & 1 group & & 2 wings & $>11$ \\
\hline Feather fan & 14 & 3 shops & 6 shops & 2 & & & $>25$ \\
\hline Specimen & 1 & & 1 shops & & & 1 & $>3$ \\
\hline Corpse & & & & & & 5 & 5 \\
\hline Living individual & & & & & & 3 & 3 \\
\hline Total & 97 & $>9$ & $>15$ & $>46$ & 1 & $>513$ & $>681$ \\
\hline
\end{tabular}

(6) Bone Flutes in literature, art and culture:

There are many articles and written references to the use of bone flutes in China. These include articles on the exploration of music and art, as well as propaganda and cultural references, eulogies, spiritual healings, religious beliefs, spiritualism, superstitions (Buddhism) and the use of totems (Xiren-Kuerban 1993). There are also references to witch culture, shamanism, intangible cultural heritage, long history stories and miraculous events (Wu 2002), which usually focus on entertainment or academic research (Wang 
2014), without any awareness of the need for protection of endangered and rare species or their conservation.

\section{A Story and Recommendation}

A recent report by Xinhua, China's largest news agency highlighted the Huaxia Ancient Music Ensemble:(http://www.xinhuanet.com/mrdx/2019$12 / 13 / \mathrm{c} . \mathrm{htm}$ ) that was set up in Henan Province 20 years ago. The ensemble use replica ancient bone flutes to play wonderful music to promote Chinese culture and perform in all parts of China and even around the world. "The sound of a bone flute is resonant and unadorned. Every time I blow into the flute, it takes me back to the mysterious ancient times," said He Xiaoshuai, a 33-year-old bone flute player of the ensemble at the Henan Museum.

When Mr. He joined the ensemble in 2006, the musicians used replicas of the Jiahu bone flutes, but the appearance, texture and tone of the replicas were nothing like the original instrument. This problem troubled them, and they decided to restore the original features of the Jiahu bone flute and allow people to hear the echoes of ancient times. The idea of finding alternative bone sources stuck with them and they sought solutions in restaurant kitchens, on river banks and with private collectors.

In 2016, a craftsman who makes Tibetan bone flutes gave to the ensemble several bones from eagles or vultures that had died of natural causes. One of the bones was similar to the Jiahu bone flute in shape and size. "It only took three to four hours to make a replica, but we spent much time over the preparation. The pitch and tone were very close to the original flute," said Mr. He, who witnessed the whole flute making process.

From this news, we can see the needs of cultural and social development, and we believe that these needs - like the ivory trade for elephants - may threaten vulture populations. Although publicizing, exhibiting, performing, selling bone flutes and trading online, are still illegal, there is some indirect evidence that villagers in rural areas obtain Griffon Vulture bones through the use of traps or poisons to supply the trade.

As a result, we put forward several suggestions for the protection of vultures:

(1) As for bone flute, we can develop simulation substitute products (Wei, 1990). For example, some people have used bone powder or plastic to make bone flutes, bone whistles and other handicrafts, as well as simulation that have successfully completed 3D designs and printing.

(2) It remains necessary to improve the legislation, modify and refine the existing wildlife protection law, and strictly prohibit the collection, production and sale of vulture bone flute and other products.

(3) Carry out scientific research and publicity activities in Xinjiang and Tibet, understand the cultural needs and survival conditions of vultures, and finally find an effective way for the protection of vultures.

\section{Acknowledgments}

The project was supported by the National Natural Science Foundation of China (31572292, 31272291, 30470262). We should especially thank to Wang Shuchao, Liu $\mathrm{Xu}, \mathrm{Xu}$ Guohua, Wu 
Daoning, Guo Hong, Zhang Yaodong, Xing Rui, Xiangchen, Lu Ping, Yimingjiang, Nie Jingbo, Huang Yahui, Chen Li (female), Ye Jin, Wang Rui, Wang Heping, Yang Feifei, Gawarxia, Shi Quan Yi, Xie Lindong, Li Zhiting, Gao Hao, Chen Xianghong, Guo Yuanqing, Chen Shengiia, Paul Xueyi, Bao Di, Lv Zhuoyi, Chai Yongbin, Niu Buzzard, Merdan Turgan, Ehlas, etc., who provide Zhongze, Yang Jun, Zhou Xiangling, Tian information and physical pictures.

Shaoxuan, Gao Shoudong, Ai Shan, Liu

\section{References}

Bower, B. 2009. Humans: Oldest known instruments found: Ivory, bone flutes date from 35,000 to 40,000 years ago. Science News 176: 13 .

Ci, D. (oral) \& Sun R.R. 2019. The eagle bone flute: an incarnation of the eagle. Tibet Geographic 19: 116-119. (In Chinese)

Duan, Y. 1995. Folk musical instruments in Gannan Tibetan Autonomous Prefecture, Gansu Province. Chinese Music 15: 48-49. (In Chinese)

Han, B.Q. 2010. On eagle flute's temperament system of Tajik nationality in China. Studies in Culture \& Art 3: 93-101. (In Chinese)

Li, S.Z. (Ming Dynasty). 1981. Compendium of Materia Medica. People's Health Press, Beijing, 2673. (In Chinese)

Ma, M., Xu, G.H., Wu, D.N. 2017. Vultures in Xinjiang. Science Press, Beijing. (In Chinese)

Ma, M. 2019. Story on Cinereous Vulture. China Forestry Publishing House, Beijing. (In Chinese)

MaMing, R., Zhao, X.M., Xu, G.H., Caiwu, J., Zhang, T., Ding, P. and Xu, F. 2014. Raptor conservation and culture in the west of China. Ela Journal 3:23-29.

MaMing, R., Xu, G.H. 2015. Status and threats to vultures in China. Vulture News 68: 3-24.

Song, S. 2007. Bone flute or bamboo flute analyze the development of civilization of the flutes. Journal of Guangxi University For Nationalities (Natural Science Edition) 13: 32-36, 44. (In Chinese)

Suo, C. 2004. On the famous Tibetan flute player--Ci Dan and his vulture bone flute. Tibetan Art Studies 19: 77-81. (In Chinese)

Wang, W. 2014. An exploration of Tajik national instrument called eagle flute. Journal of Kashgar University 35: 48-50, 81. (In Chinese) DOI: 10.3969/j.issn.1006-432X.2014.05.012

Wang, Z.C. 2001. Bone flute, bone whistle (2). Musical Instruments Magazine 49: 92-93. (In Chinese)

Wei, Z.K. 1990. Imitation bone flute of Hemudu and passed technical appraisal in Yuyao. Musical Instrument Magazine 19: 20. (In Chinese) 
Wu, G.D. 2002. Four points about historical pictures--The related description of bone whistle, bone flute, earthen bell and crocodile skin drum picture. Journal of Nanyang Teachers' College (Social Sciences) 1: 68-71. (In Chinese)

Xiren-Kuerban. 1993. Eagle and Tajik culture. Journal of Xinjiang University 21: 75-80. (In Chinese)

Xue, H.P. 2010. Application and characteristics of vulture bone flute with Tibetan life in Qinghai Province. National Music 46: 8-9. (In Chinese)

Zhang, D.B. 1990. Research on the origin and development of Qiang flute. Journal of Qinghai Normal University (Philosophy and Social Sciences Edition) 3: 84-90. (In Chinese)

Zhang, J.Z., Harbottle, G., Wang, C., Kong, Z. 1999. Oldest playable musical instruments found at Jiahu early Neolithic site in China. Nature 401: 366-368.

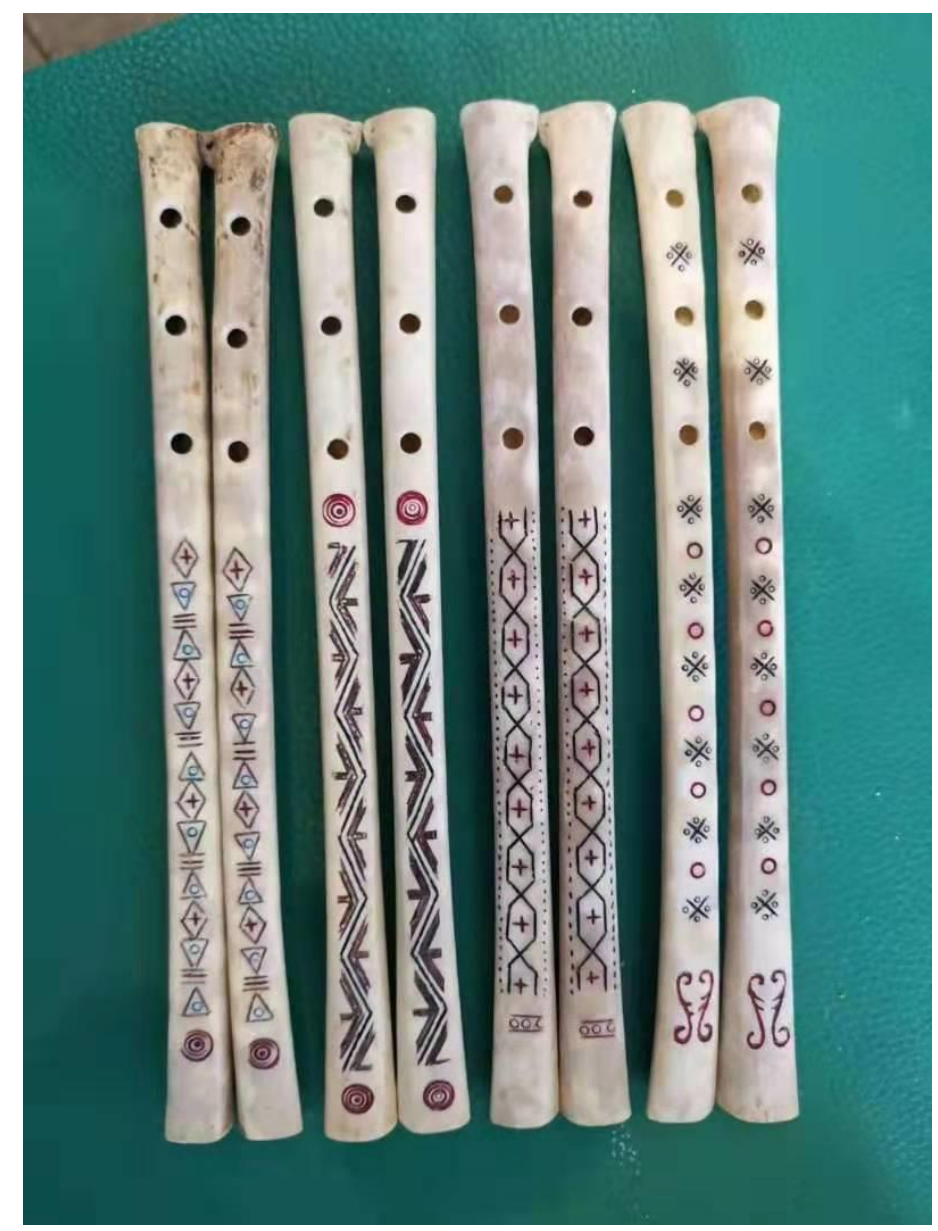

Three hole vulture bone flutes of Tajik nationality, Xinjiang, photographed by Gao Hao, May 2018 


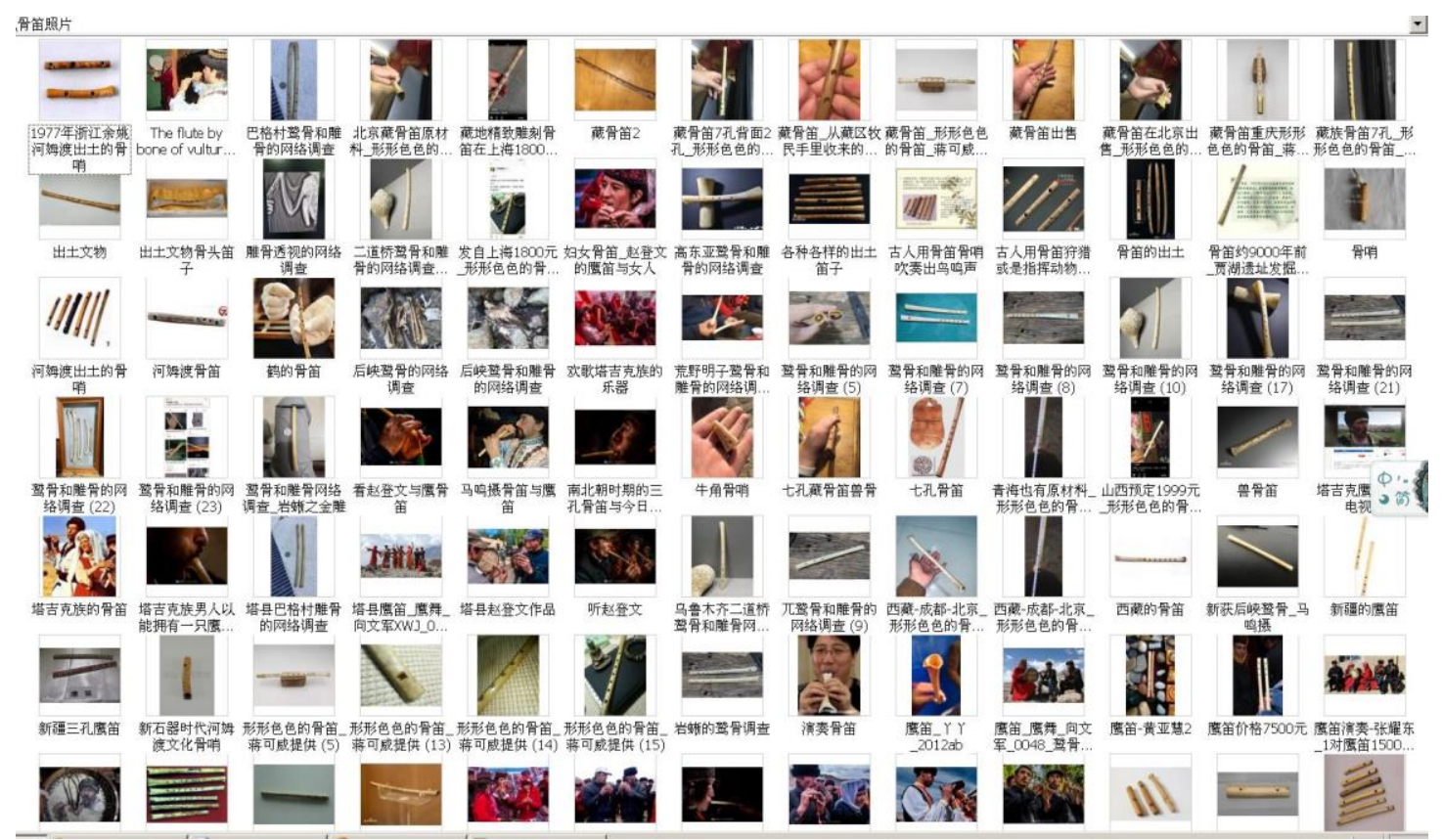

Vulture bone flutes available for sale on the internet in China

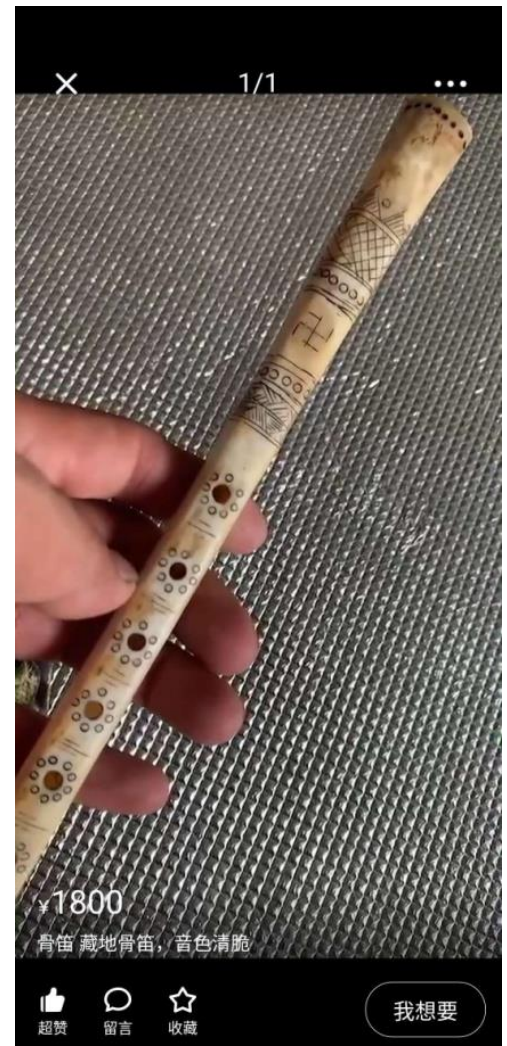

Tibet style beautiful 6-hole vulture bone flute sold on the internet (Jang Kewei) 


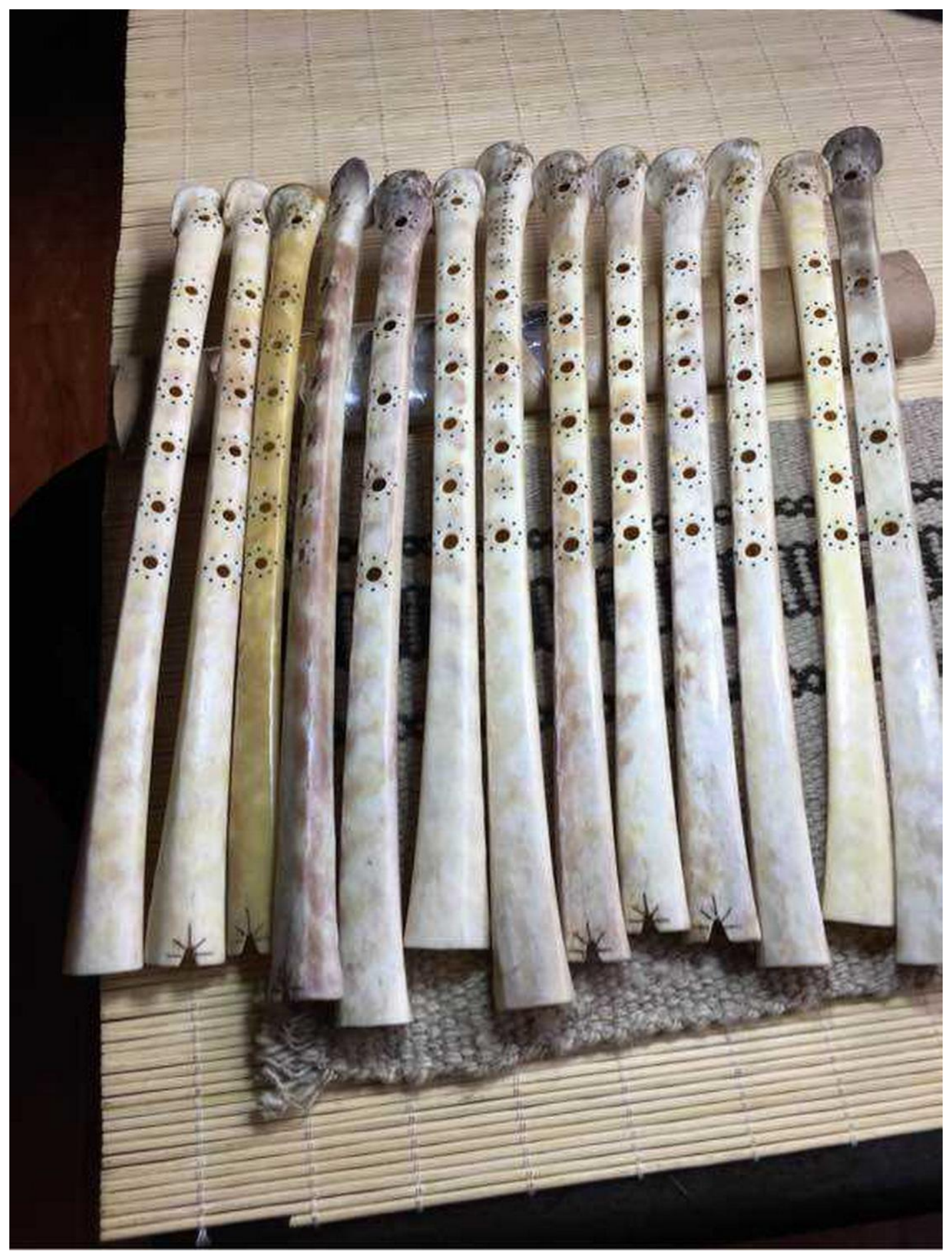

Tibet vulture bone flutes with 6-hole (Jiang Kewei) 


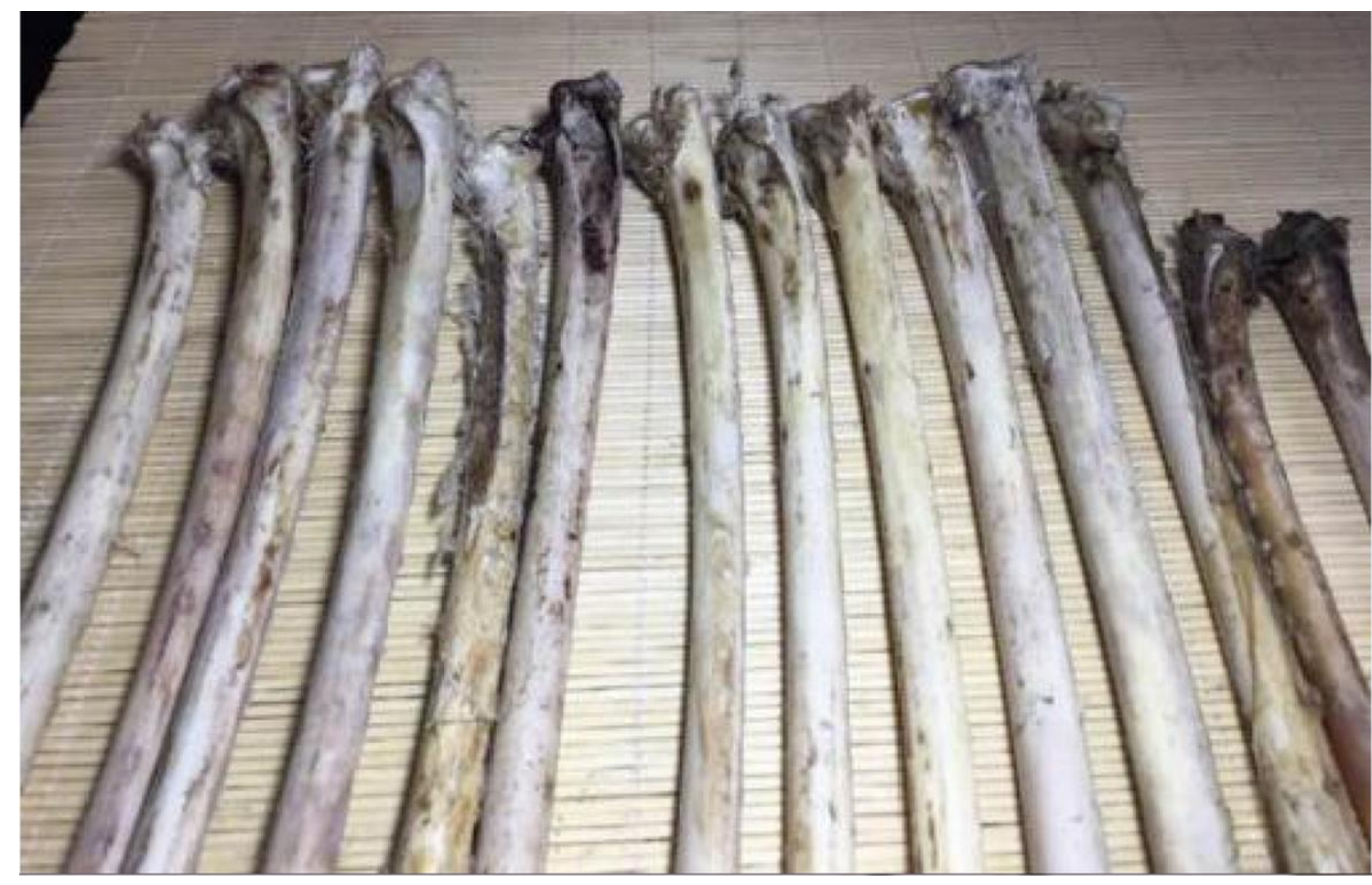

Vulture bone materials from Tian Xiangdong_2019

$* * * * * *$ 